\title{
Majorization of sequences, sharp vector Khinchin inequalities, and bisubharmonic functions
}

by

\section{Albert Baernstein II and Robert C. Culverhouse (St. Louis, MO)}

\begin{abstract}
Let $X=\sum_{i=1}^{k} a_{i} U_{i}, Y=\sum_{i=1}^{k} b_{i} U_{i}$, where the $U_{i}$ are independent random vectors, each uniformly distributed on the unit sphere in $\mathbb{R}^{n}$, and $a_{i}, b_{i}$ are real constants. We prove that if $\left\{b_{i}^{2}\right\}$ is majorized by $\left\{a_{i}^{2}\right\}$ in the sense of Hardy-Littlewood-Pólya, and if $\Phi: \mathbb{R}^{n} \rightarrow \mathbb{R}$ is continuous and bisubharmonic, then $E \Phi(X) \leq E \Phi(Y)$. Consequences include most of the known sharp $L^{2}-L^{p}$ Khinchin inequalities for sums of the form $X$. For radial $\Phi$, bisubharmonicity is necessary as well as sufficient for the majorization inequality to always hold. Counterparts to the majorization inequality exist when the $U_{i}$ are uniformly distributed on the unit ball of $\mathbb{R}^{n}$ instead of on the unit sphere.
\end{abstract}

1. Main results. In this first section, we set up notation and state our main results, about sums of independent random vectors uniformly distributed on spheres in $\mathbb{R}^{n}$. History and background are provided in $\S 2$, Theorems $1,2,3$ are proved in $\S 3,4,5$, and $\S 6$ contains a formula which permits transfer of the results in $\S 1$ to sums of independent random vectors uniformly distributed on balls in $\mathbb{R}^{n}$.

Throughout the paper, $a=\left(a_{1}, \ldots, a_{k}\right)$ and $b=\left(b_{1}, \ldots, b_{k}\right)$ will denote $k$-tuples of real numbers. Following the terminology of [MO 79], we say that $a$ is majorized by $b$, and write $a \prec b$, or $\left\{a_{i}\right\} \prec\left\{b_{i}\right\}$, if

$$
\sum_{i=1}^{k} a_{i}=\sum_{i=1}^{k} b_{i}, \quad \text { and } \quad \sum_{i=1}^{j} a_{i}^{*} \leq \sum_{i=1}^{j} b_{i}^{*}, \quad j=1, \ldots, k,
$$

where $\left\{a_{i}^{*}\right\}$ denotes the sequence $\left\{a_{i}\right\}$ rearranged in decreasing order.

Let $\Delta$ denote the Laplace operator in $\mathbb{R}^{n}$. A continuous function $\Phi$ : $\mathbb{R}^{n} \rightarrow \mathbb{R}$ is said to be bisubharmonic in $\mathbb{R}^{n}$ if the distribution $\Delta \Delta \Phi$ equals

2000 Mathematics Subject Classification: Primary 60E15; Secondary 60G50.

The first author was supported in part by NSF grants DMS-9501293 and DMS9801282. The second author was supported by NSF grant DMS-9501293 and NIMH grant T32-MHI4677. 
a positive Radon measure on $\mathbb{R}^{n}$. Equivalently, $\Phi$ is bisubharmonic if the distribution $\Delta \Phi$ equals a subharmonic function on $\mathbb{R}^{n}$, in the sense of distributions. If $\Phi \in C^{4}\left(\mathbb{R}^{n}, \mathbb{R}\right)$, then $\Phi$ is bisubharmonic if and only if $\Delta \Delta \Phi \geq 0$ at every point in $\mathbb{R}^{n}$. A function is said to be bisuperharmonic if its negative is bisubharmonic.

For $n \geq 1$, let $U_{1}, \ldots, U_{k}$ be independent $\mathbb{R}^{n}$-valued random variables defined on some probability space, each of which is uniformly distributed on the unit sphere $S^{n-1}=\left\{x \in \mathbb{R}^{n}:|x|=1\right\} \subset \mathbb{R}^{n}$. We use $|x|$ to denote the Euclidean norm of $x$. When $n=1$, the $U_{i}$ take on each of \pm 1 with probability $1 / 2$ and are called symmetric Bernoulli random variables. Set, for general $n$,

$$
X=\sum_{i=1}^{k} a_{i} U_{i}, \quad Y=\sum_{i=1}^{k} b_{i} U_{i} .
$$

THEOREM 1. Let $\Phi$ be bisubharmonic and continuous on $\mathbb{R}^{n}$. If $\left\{b_{i}^{2}\right\} \prec$ $\left\{a_{i}^{2}\right\}$, then

$$
E \Phi(X) \leq E \Phi(Y) .
$$

If $\Phi$ is bisuperharmonic and continuous on $\mathbb{R}^{n}$, then Theorem 1 implies that the inequality in (1.1) reverses. The message of the theorem is that spreading out the coefficients in the sum defining $X$, while keeping their sum of squares constant, increases the $\Phi$ moment, when $\Phi$ is bisubharmonic. A counterpart of Theorem 1 for balls will be discussed in $\S 6$.

In case $\Phi$ is radial, that is, $\Phi(x)$ depends only on $|x|$, Theorem 1 admits a converse:

TheOREM 2. Suppose that $\Phi: \mathbb{R}^{n} \rightarrow \mathbb{R}$ is continuous and radial. If (1.1) holds for every pair of $k$-tuples $a, b$ with $\left\{b_{i}^{2}\right\} \prec\left\{a_{i}^{2}\right\}$ and every $k \geq 2$, then $\Phi$ is bisubharmonic in $\mathbb{R}^{n}$.

For any $\Phi \in C\left(\mathbb{R}^{n}, \mathbb{R}\right)$, let $\Phi_{1}$ be the radial function on $\mathbb{R}^{n}$ obtained by setting $\Phi_{1}(x)$ equal to the mean value of $\Phi$ on the sphere $|x| S^{n-1}$. We will call $\Phi_{1}$ the radialization of $\Phi$. Since the distribution of $X$ is invariant under rotations, we have $E \Phi(X)=E \Phi_{1}(X)$. Moreover, if $\Phi$ is continuous and bisubharmonic, then so is $\Phi_{1}$. Using these facts, one sees that Theorems 1 and 2 are equivalent to the following "if and only if" statement:

$$
\begin{aligned}
E \Phi(X) \leq E \Phi(Y) \text { for every } a, b \text { with }\left\{b_{i}^{2}\right\} \prec & \left\{a_{i}^{2}\right\} \\
& \Leftrightarrow \Phi_{1} \text { is bisubharmonic. }
\end{aligned}
$$

The statement above characterizes continuous functions $\Phi$ for which a majorization inequality holds for $\Phi$ moments. The next statement, a close neighbor to Theorems 1 and 2, gives a characterization of continuous functions which are bisubharmonic. 
TheOREm 3. Let $\Phi: \mathbb{R}^{n} \rightarrow \mathbb{R}$ be continuous. Then $\Phi$ is bisubharmonic in $\mathbb{R}^{n}$ if and only if the function

$$
t \mapsto \int_{S^{n-1}} \Phi\left(x_{0}+t^{1 / 2} x\right) d x
$$

is convex on $(0, \infty)$ for every $x_{0} \in \mathbb{R}^{n}$.

Convention. In integrals over $S^{n-1}, d x$ will denote the uniform probability measure on $S^{n-1}$. In integrals over the unit ball $\mathbb{B}^{n} \subset \mathbb{R}^{n}, d x$ will denote the uniform probability measure on $\mathbb{B}^{n}$.

For each $k$-tuple $a$ with $\sum_{i=1}^{k} a_{i}^{2}=1$ we have $\left(k^{-1}, \ldots, k^{-1}\right) \prec\left\{a_{i}^{2}\right\} \prec$ $(1,0, \ldots, 0)$, where the first and third sequences are $k$-tuples. Moreover, the $(k+1)$-tuple $\left((k+1)^{-1}, \ldots,(k+1)^{-1}\right)$ is majorized by the $(k+1)$-tuple $\left(k^{-1}, \ldots, k^{-1}, 0\right)$. For $n \geq 1, k \geq 1$, define

$$
Z_{n, k}=k^{-1 / 2} \sum_{i=1}^{k} U_{i},
$$

and define $Z_{n}$ to be the $\mathbb{R}^{n}$-valued random variable whose components are independent mean-zero real normal random variables, each with variance $1 / n$. Let $\Phi$ be continuous and bisubharmonic. Then Theorem 1 implies that $E \Phi\left(Z_{n, k}\right)$ increases as $k$ increases when $n$ is fixed. In $\S 3$, we will show that the radialization $\Phi_{1}$ of $\Phi$ satisfies

$$
E\left(\left(\Phi_{1}\left(Z_{n}\right)\right)^{-}\right)<\infty
$$

so that $E \Phi_{1}\left(Z_{n}\right)$ is well defined, and that

$$
\lim _{k \rightarrow \infty} E \Phi\left(Z_{n, k}\right)=E \Phi_{1}\left(Z_{n}\right) .
$$

Note that for $X=\sum_{i=1}^{k} a_{i} U_{i}$, we have $E|X|^{2}=\sum_{i=1}^{k} a_{i}^{2}$. In particular, $E\left|Z_{n, k}\right|^{2}=E\left|U_{1}\right|^{2}=1$. For fixed $n$ and appropriate $\Phi$, the corollaries to follow assert two things: (a) Among all $X$ with fixed $L^{2}$ norm and at most $k$ summands, the extremal $\Phi$ moments are achieved by multiples of $U_{1}$ and $Z_{n, k}$. (b) Among all $X$ with fixed $L^{2}$ norm, the supremum and infimum of the $\Phi$ moments are furnished by multiples of $U_{1}$ and $Z_{n}$.

COROLlaRY 1. If $\Phi$ is continuous and bisubharmonic on $\mathbb{R}^{n}$ and $E|X|^{2}$ $=1$, then

$$
E \Phi\left(U_{1}\right) \leq E \Phi(X) \leq E \Phi\left(Z_{n, k}\right) \leq E \Phi_{1}\left(Z_{n}\right) .
$$

The quantities on the right and left ends are the best possible constants which work simultaneously for all $k$ and fixed $n$.

The inequalities follow from Theorem 1 and (1.3). The left-hand constant is obviously optimal; optimality of the right-hand constant follows 
from (1.3). The right-hand constant is sometimes $\infty$. But if it is finite, then $E\left|\Phi\left(Z_{n}\right)\right|$ is also finite, and $E \Phi_{1}\left(Z_{n}\right)=E \Phi\left(Z_{n}\right)$.

Of course, one could replace the hypothesis $E|X|^{2}=1$ with $E|X|^{2}=$ some fixed number $M^{2}$, provided a factor $M$ is inserted at appropriate places in the inequalities.

Let us consider the $p$ th moment case: $\Phi(x)=|x|^{p}, p>0$. For radial $\Phi$ on $\mathbb{R}^{n}$, we have $\Delta \Phi=\Phi_{r r}+((n-1) / r) \Phi_{r}$, where the subscript $r$ denotes radial differentiation. Thus, for $x \in \mathbb{R}^{n} \backslash\{0\}$,

$$
\begin{aligned}
\Delta|x|^{p} & =p(p+n-2)|x|^{p-2}, \\
\Delta \Delta|x|^{p} & =p(p+n-2)(p-2)(p+n-4)|x|^{p-4} .
\end{aligned}
$$

The task of deciding which functions $|x|^{p}$ are bisub- or bisuperharmonic on all of $\mathbb{R}^{n}$ is complicated by the singularity at the origin. One way to overcome the difficulty is to consider the functions $u_{\varepsilon}(x)=\left(|x|^{2}+\varepsilon\right)^{p / 2}$. Then $u_{\varepsilon} \in C^{\infty}\left(\mathbb{R}^{n}\right)$ for $\varepsilon>0, p>0$, and for $x \in \mathbb{R}^{n}$,

$$
\Delta u_{\varepsilon}(x)=p(p-2)\left(r^{2}+\varepsilon\right)^{p / 2-4}\left(A r^{4}+B r^{2}+C\right),
$$

where $r=|x|$ and

$A=(p+n-2)(p+n-4), \quad B=2 \varepsilon(n+2)(p+n-4), \quad C=\varepsilon^{2} n(n+2)$.

We deduce that, when $n=1, u_{\varepsilon}$ is bisubharmonic for $p \geq 3$; when $n \geq 2, u_{\varepsilon}$ is bisubharmonic for $p \geq 2$; when $n=3, u_{\varepsilon}$ is bisuperharmonic for $1 \leq p \leq 2$; and when $n \geq 4, u_{\varepsilon}$ is bisuperharmonic for $0<p \leq 2$. Since $u_{\varepsilon} \rightarrow|x|^{p}$ uniformly on compact subsets of $\mathbb{R}^{n}$ as $\varepsilon \rightarrow 0$, it follows that $|x|^{p}$ is bisub- or bisuperharmonic for the same range of $p$ and $n$. From Corollary 1 we obtain

Corollary 2. Let $X$ be as in Corollary 1 . If $p \geq 3$ when $n=1$, or if $p \geq 2$ when $n \geq 2$, then

$$
1 \leq E|X|^{p} \leq E\left|Z_{n, k}\right|^{p} \leq E\left|Z_{n}\right|^{p}=\frac{\Gamma((p+n) / 2)}{\Gamma(n / 2)} 2^{p / 2} n^{-p / 2} .
$$

Moreover, when $n=3$ and $1 \leq p \leq 2$ all the inequalities reverse, and when $n \geq 4$ and $0<p \leq 2$ all the inequalities reverse. In each of the stated cases for $n$ and $p$, the quantities on the right and left ends are the best possible constants which work simultaneously for all $k$ and fixed $n$.

For $p$ and $n$ not covered by Corollary $2,|x|^{p}$ is neither bisub- nor bisuperharmonic on $\mathbb{R}^{n}$. Consider, for example, $n=2$ and $0<p<2$. One can show that the distributional Laplacian of $|x|^{p}$ on $\mathbb{R}^{2}$ is the locally integrable function $p^{2}|x|^{p-2}$. This function is subharmonic in $\mathbb{R}^{2} \backslash\{0\}$, but is not a.e. equal to a subharmonic function in $\mathbb{R}^{2}$, since its limit as $x \rightarrow 0$ is $\infty$. Thus, $|x|^{p}$ is neither bisub- nor bisuperharmonic on $\mathbb{R}^{2}$. 
Corollary 1 also produces sharp logarithmic Khinchin inequalities, provided the dimension $n$ is sufficiently large. For $x \in \mathbb{R}^{n} \backslash\{0\}$ and $r=|x|$, the reader is invited to verify that

$$
\Delta \log (1+|x|)=\frac{n-1+(n-2) r}{r(1+r)^{2}}
$$

and that

$$
r^{3}(1+r)^{4} \Delta \Delta \log (1+|x|)=-\sum_{j=0}^{3} c_{j} r^{j}
$$

where

$$
\begin{array}{ll}
c_{0}=(n-1)(n-3), & c_{1}=4(n-1)(n-3), \\
c_{2}=(n-1)(5 n-19), & c_{3}=2(n-2)(n-4) .
\end{array}
$$

If $n \geq 4$ then all the $c_{i}$ are nonnegative. Thus, $\log (1+|x|)$ is bisuperharmonic in $\mathbb{R}^{n} \backslash\{0\}$. By an approximation argument like the one for $p$ th moments, or by other means, one can show that $\log (1+|x|)$ is bisuperharmonic on all of $\mathbb{R}^{n}$. The same is true for $\log (A+|x|)$ for each nonnegative constant $A$. Corollary 1 implies

Corollary 3. Let $X$ be as in Corollary 1 , and $n \geq 4$. Then for every nonnegative constant $A$,

$$
\begin{aligned}
E \log \left(A+\left|Z_{n}\right|\right) & \leq E \log \left(A+\left|Z_{n, k}\right|\right) \\
& \leq E \log (A+|X|) \leq \log (A+1) .
\end{aligned}
$$

The quantities on the right and left ends are the best possible constants which work simultaneously for all $k$ and fixed $n \geq 4$.

2. A brief history of sharp Khinchin inequalities. First, we review work in dimension $n=1$, so that $X=\sum_{i=1}^{k} a_{i} U_{i}$ is a real linear combination of symmetric Bernoulli random variables. We remind the reader that $Z_{1}$ denotes a real-valued standard normal random variable, and that

$$
Z_{1, k}=k^{-1 / 2} \sum_{i=1}^{k} U_{i} .
$$

In this whole section, we shall always assume the $L^{2}$-normalization

$$
E|X|^{2}=\sum_{i=1}^{k} a_{i}^{2}=1
$$

Khinchin [Kh 23] proved existence of constants $A_{p}$ and $B_{p}, 0<p<\infty$, such that $A_{p} \leq E|X|^{p} \leq B_{p}$. We shall denote the best such constants also by $A_{p}, B_{p}$, and call them the best $L^{2}-L^{p}$ constants. Hölder's inequality implies 
that $B_{p}=1$ for $0<p \leq 2$ and $A_{p}=1$ for $2 \leq p<\infty$. The first nontrivial best constants were found by Whittle [W 60], who asserted that $B_{p}=E\left|Z_{1}\right|^{p}$ for $2<p<\infty$. However, as pointed out in [F 97, p. 998], Whittle's proof is valid only for $3 \leq p<\infty$. The "Gaussian bound" $B_{p}=E\left|Z_{1}\right|^{p}, p \geq 3$, was proved again by Young [Y 76]. Earlier, Stechkin [Ste 61] had proved $B_{2 m}=E\left|Z_{1}\right|^{2 m}$ for positive integers $m$. Presumably, Young and Stechkin were unaware of Whittle's paper. Szarek [Sz 76] proved that $A_{p}=E\left|Z_{1}\right|^{p}$ for $p=1$ or $p$ slightly larger than 1 .

To find the remaining best constants turned out to be much more difficult. This feat was accomplished by Haagerup [Ha 82], who proved that $B_{p}=E\left|Z_{1}\right|^{p}$ for all $p>2$, while for $0<p<2, A_{p}$ is the smaller of $E\left|Z_{1}\right|^{p}$ and $E\left|Z_{1,2}\right|^{p}$. Letting $p_{1} \approx 1.8$ be the unique solution $p \in(0,2)$ to the equation $\Gamma\left(\frac{1}{2}(p+1)\right)=\frac{1}{2} \sqrt{\pi}$, the smaller is $E\left|Z_{1}\right|^{p}$ when $p_{1}<p<2$, and is $E\left|Z_{1,2}\right|^{p}$ when $0<p<p_{1}$.

Haagerup's proof requires lots of integrals and estimates. An ingenious alternative proof for $0<p<2$, shorter but still complicated, can be found in [NP 96].

Turning now to majorization inequalities, still in dimension $n=1$, set $Y=\sum_{i=1}^{k} b_{i} U_{i}$. Eaton [E 70] proved that $\left\{b_{i}^{2}\right\} \prec\left\{a_{i}^{2}\right\} \Rightarrow E \Phi(X) \leq E \Phi(Y)$ for functions $\Phi: \mathbb{R} \rightarrow \mathbb{R}$ which satisfy a certain condition. Komorowski [Kom 88] showed that Eaton's condition is satisfied by $K(x)=|x|^{p}$ when $p \geq 3$, and thereby obtained the $n=1$ case of our Corollary 2 .

It turns out that Eaton's condition on $\Phi$ is in fact equivalent to convexity of $\Phi^{\prime \prime}$. Thus, after tending to a few technicalities, one sees that Eaton's Theorem coincides with our Theorem 1 for $n=1$.

When $n=2$ the $U_{i}$ are uniformly distributed on the unit circle $S^{1}$, and are sometimes called Steinhaus random variables. Suppressing their dependence on the dimension, we continue to denote the corresponding best $L^{2}-L^{p}$ Khinchin constants by $A_{p}$ and $B_{p}$. The trivial bounds $A_{p}=1$ for $p \geq 2$, $B_{p}=1$ for $p \leq 2$ are still true. Haagerup (see [Pe 85, p. 151]) conjectured that $B_{p}=E\left|Z_{2}\right|^{p}$ for $p>2$, and $A_{p}=\min \left(E\left|Z_{2}\right|^{p}, E\left|Z_{2,2}\right|^{p}\right)$ for $0<p<2$. Here, for $0<p<2$, the Gaussian is smaller if and only if $p_{2}<p<2$, where $p_{2} \approx 0.48$. Haagerup's conjecture was verified by Sawa [S 85] for $p=1$ or $p$ close to 1 . Sawa stated that he could prove $A_{p}=E\left|Z_{2}\right|^{p}$ for every $p \in\left[p_{2}, 2\right)$ and $B_{p}=E\left|Z_{2}\right|^{p}$ for every $p>2$, but the proofs were never published.

Peškir [Peš 95] proved that when $n=2$ one has the majorization inequality $\left\{b_{i}^{2}\right\} \prec\left\{a_{i}^{2}\right\} \Rightarrow E|X|^{2 m} \leq E|Y|^{2 m}$ for positive integers $m$. A consequence is that $B_{2 m}=E\left|Z_{2}\right|^{2 m}$ when $n=2$. König [Kö 98] and Culverhouse [C 98] independently confirmed that $B_{p}=E\left|Z_{2}\right|^{p}$ for every $p>2$. In fact, they proved that the $n$-dimensional best $L^{2}-L^{p}$ Khinchin constant $B_{p}$ is $B_{p}=E\left|Z_{n}\right|^{p}$ for every $p>2$ and $n \geq 2$. In addition, König proved that the best $n$-dimensional constant $A_{p}$ satisfies $A_{p}=E\left|Z_{n}\right|^{p}$ for $1 \leq p<2$ 
when $n \geq 2$, and for $0<p<2$ when $n \geq 3$. All of these results follow from Theorem 1 and are included in Corollary 2 of the present paper, except for $1 \leq p<2$ when $n=2$, and $0<p<1$ when $n=3$.

For each $n \geq 1$ and $p \geq 3$, the bound $B_{p}=E\left|Z_{n}\right|^{p}$ can also be obtained by clever application of Theorem 1 in [F 97]. We thank S. Kwapień for pointing this out to us.

Let $V_{1}, \ldots, V_{k}$ be independent random variables, each of which is uniformly distributed on the unit interval $[-1,1]$. Latała and Oleszkiewicz [LO 95] proved that

$$
\left\{b_{i}^{2}\right\} \prec\left\{a_{i}\right\}^{2} \Rightarrow E\left|\sum_{i=1}^{k} a_{i} V_{i}\right|^{p} \leq E\left|\sum_{i=1}^{k} b_{i} V_{i}\right|^{p}
$$

for $2 \leq p<\infty$, while for $1 \leq p \leq 2$ the inequality reverses. As with spheres, the majorization inequalities permit identification of the corresponding best Khinchin $L^{2}-L^{p}$ constants for random variables of the form $\sum_{i=1}^{k} a_{i} V_{i}$. Culverhouse [C 98], building on ideas in [LO 95], obtained the analogues of (2.1) and its reverse inequality for $V_{i}$ uniformly distributed on balls of any dimension when $1 \leq p<\infty$. [C 98] also contains majorization inequalities for $\Phi\left(\sum_{i=1}^{k} a_{i} V_{i}\right)$ and $\Phi\left(\sum_{i=1}^{k} b_{i} V_{i}\right)$ for some more general $\Phi$, and the $S^{n-1}$-analogue of the majorization inequality (2.1) for $n \geq 2$ and $p \geq 2$.

The proofs in [C 98] required power series computations which were pleasant for $n=2$ but quite taxing for $n \geq 3$. Accordingly, the computations for $n \geq 3$ were omitted from the thesis [C 98], the intention being to include them in a subsequent paper based on the thesis. Meanwhile, we heard from Professor König about his work showing equivalence of ball and sphere problems, and this inspired us to take a fresh look at all the results in [C 98]. Eventually, we found the tie between Khinchin-type inequalities and bisubharmonic functions embodied in Theorems 1-3. The present Theorem1 and its ball counterpart - stated in $\S 6$ - contain results more general than those stated in [C 98], and are proved with much less effort.

For $\Phi(x)=|x|^{p}$ and $p$ and $n$ as in Corollary 2, the $L^{p}$ majorization inequalities supplied by our Theorem 1 are proved also in [KK 01], via a somewhat different path. See Remark (ii), p. 122 of [KK 01]. A key element of the KK proofs is convexity of the function $t \mapsto E\left|x_{0}+t^{1 / 2} U\right|^{p}$ for appropriate $p$ and $n$, where $U$ is uniformly distributed on $S^{n-1}$. It was after seeing this that we were led to formulate our Theorem 3 and Lemma 1. (We had already proved Theorems 1 and 2, by arguments less simple than those used here.) The KK proof of convexity makes use of heavy-duty one- and two-variable calculus, whereas our more general Theorem 3 uses only lightduty $n$-variable calculus: an application of the divergence theorem to the appropriate integral proves the convexity almost immediately. 
We remark also that Theorem 1 can be derived by combining Theorem 3 with some arguments in [KK 01] or [LO 95], but we have opted to give a selfcontained proof of Theorem 1, to highlight the salutary role the divergence theorem can play in the study of vector Khinchin inequalities.

To recapitulate: On spheres $S^{n-1}$, sharp $L^{2}-L^{p}$ Khinchin inequalities, and much more, can be painlessly proved when $n=1$ and $p \geq 3$, when $n=2$ and $p \geq 2$, when $n=3$ and $p \geq 1$, and when $n=4$ and $p>0$ (Theorem 1 , Corollary 2). With vigorous hard analysis, sharp Khinchin inequalities have also been proved when $n=1$ and $0<p<2$ or $2<p<3$, when $n=2$ and $1 \leq p<2$, and when $n=3$ and $0<p<1$ ([Ha 82], [Kö 98], [KK 01]). To illustrate the difficulty gap between the two sets of results, one may note that KK prove their results which overlap ours within a space of about 12 pages, but require approximately 25 pages full of estimates and integrals of Bessel functions and other quantites to dispose of $n=2, p \in[1,2)$.

For $n=2$ and $0<p<1$, Haagerup's conjecture for the best $A_{p}$ remains open, except for $p$ close enough to 1 so that Sawa's proof is valid. The logarithmic counterpart to Haagerup's Conjecture seems especially interesting: When $n=2$, is it true that

$$
E \log |X| \geq E \log \left|Z_{1,2}\right| ?
$$

With some absolute constant on the right, (2.2) was proved, independently, by Favorov [Fa 87] and Ullrich [U 88]. Gorin and Favorov [GF 87] (see also [Fa 98]) generalized this result by proving $n$-dimensional inequalities for negative moments: $E|X|^{-p} \leq C_{p, n}$ when $n \geq 2$ and $0<p<n-1$. As far as we know, the best constants $C_{p, n}$ are not known in any dimension. On the other hand, our Corollary 3 shows that if $n \geq 4$ the best constant on the right in (2.2) is $E \log \left|Z_{n}\right|$.

There remain some other open questions about which of the inequalities in Theorem 1 and Corollary 2 continue to hold when $n$ and $p$ do not satisfy the hypotheses of Corollary 2. We will forgo a systematic discussion, but will present two examples which furnish negative results.

Example 1. Take $n=1$. The function $\psi(p)=4 p-5-3^{p-1}$ is concave and has two zeros on $\mathbb{R}$, at $p=2$ and $p=p^{*}$, where $2.34<p^{*}<2.35$. For each $p \in\left(2, p^{*}\right)$, there exists $\varepsilon_{p}>0$ such that if $X=\sum_{i=1}^{3} a_{i} U_{i}$, with $a_{1}=(2 / 3)^{1 / 2} \cos \theta, a_{2}=(2 / 3)^{1 / 2} \sin \theta, a_{3}=3^{-1 / 2}$, and $|\theta-\pi / 4|<\varepsilon_{p}$, then $E|X|^{p}>E\left|Z_{1,3}\right|^{p}$. Thus, the second inequality in (1.6) can fail when $2<p<p^{*}$, whereas $E|X|^{p} \leq E\left|Z_{1}\right|^{p}$, by Haagerup's Theorem.

EXAmPle 2. Take $n=2$. For $\delta>0$, set

$$
X=U_{1}+\delta U_{3}, \quad Y=U_{1}+\frac{1}{\sqrt{2}} \delta U_{2}+\frac{1}{\sqrt{2}} \delta U_{3} .
$$


For $p>0$ write $\alpha=p / 2$. Then, as $\delta \rightarrow 0$,

$$
\begin{aligned}
& E|X|^{p}=1+\alpha^{2} \delta^{2}+\frac{\alpha^{2}(\alpha-1)^{2}}{4} \delta^{4}+O\left(\delta^{6}\right), \\
& E|Y|^{p}=1+\alpha^{2} \delta^{2}+\frac{3}{2} \frac{\alpha^{2}(\alpha-1)^{2}}{4} \delta^{4}+O\left(\delta^{6}\right) .
\end{aligned}
$$

Thus, $E|X|^{p}<E|Y|^{p}$ for sufficiently small $\delta$ and all $p$ except $p=2$. In particular, a conceivable strengthening of Haagerup's Conjecture,

$$
\left\{b_{i}^{2}\right\} \prec\left\{a_{i}^{2}\right\} \Rightarrow E|Y|^{p} \leq E|X|^{p}, p \in\left[p_{2}, 2\right),
$$

is false for every $p \in\left[p_{2}, 2\right)$.

3. Proof of Theorem 1. For continuous $f \in C\left(\mathbb{R}^{n}, \mathbb{R}\right)$, set

$$
I(r)=I(r, f)=\int_{S^{n-1}} f(r x) d x, \quad r \geq 0 .
$$

We remind the reader of our convention that in integrals over $S^{n-1}, d x$ denotes normalized uniform measure on $S^{n-1}$, and that in integrals over unit balls $\mathbb{B}^{n}=\left\{x \in \mathbb{R}^{n}:|x|<1\right\}, d x$ denotes normalized Lebesgue measure on $\mathbb{B}^{n}$.

The proof of Theorem 1 involves mostly elementary integral identities, two of which are stated in the following lemmas.

Lemma 1. Suppose that $f \in C^{2}\left(\mathbb{R}^{n}, \mathbb{R}\right)$. Then

$$
\frac{d}{d t} I\left(t^{1 / 2}, f\right)=\frac{1}{2 n} \int_{\mathbb{B}^{n}} \Delta f\left(t^{1 / 2} x\right) d x, \quad 0 \leq t<\infty .
$$

Proof. An argument using the divergence theorem, and the relation $s=$ $n b$ between the unnormalized measures $s$ of $S^{n-1}$ and $b$ of $\mathbb{B}^{n}$, gives

$$
I^{\prime}(r)=\frac{r}{n} \int_{\mathbb{B}^{n}} \Delta f(r x) d x
$$

from which (3.2) follows.

In the next lemma, $e_{1}$ denotes the unit vector $(1,0, \ldots) \in \mathbb{R}^{n}$.

Lemma 2. Suppose that $f \in C\left(\mathbb{R}^{n}, \mathbb{R}\right)$. Then, for $\alpha, \beta \in \mathbb{R}$,

$$
\int_{\left(S^{n-1}\right)^{2}} f(\alpha x+\beta y) d x d y=\int_{S^{n-1}} I\left(\left|\alpha e_{1}+\beta y\right|, f\right) d y .
$$

Proof. Fix $n \geq 2$, and write $S=S^{n-1}$. Let $G$ denote the special orthogonal group $\mathrm{SO}(n)$, and $d g$ denote normalized Haar measure on $G$. Then for continuous functions $F$ on $\mathbb{R}^{n}$ and $z \in \mathbb{R}^{n}$, we have $\int_{G} F(g z) d g=$ 
$\int_{S} F(|z| x) d x$, where $g z$ denotes the action of $g$ on $z$. Thus

$$
\begin{aligned}
\int_{S \times S} f(\alpha x+\beta y) d x d y & =\int_{G \times G} f\left(\alpha g_{1} e_{1}+\beta g_{2} e_{1}\right) d g_{1} d g_{2} \\
& =\int_{G} d g_{1} \int_{G} f\left(g_{1}\left(\alpha e_{1}+\beta g_{1}^{-1} g_{2} e_{1}\right)\right) d g_{2} \\
& =\int_{G} d g_{1} \int_{G} f\left(g_{1}\left(\alpha e_{1}+\beta g_{2} e_{1}\right)\right) d g_{2} \\
& =\int_{G} d g_{2} \int_{G} f\left(g_{1}\left(\alpha e_{1}+\beta g_{2} e_{1}\right)\right) d g_{1} \\
& =\int_{G} d g_{2} \int_{S} f\left(\left|\alpha e_{1}+\beta g_{2} e_{1}\right| y\right) d y \\
& =\int_{G} I\left(\left|\alpha e_{1}+\beta g_{2} e_{1}\right|, f\right) d g_{2} \\
& =\int_{S} I\left(\left|\alpha e_{1}+\beta y\right|, f\right) d y .
\end{aligned}
$$

Proof of Theorem 1. We may assume that each of the $a_{i}$ and each of the $b_{i}$ are nonnegative, and, via the usual approximation arguments, that the bisubharmonic function $\Phi$ is in $C^{4}$. Let $\mathbb{R}_{+}^{k}$ be the set of all points $s=\left(s_{1}, \ldots, s_{k}\right) \in \mathbb{R}^{k}$ with each $s_{i} \geq 0$. Define $Q: \mathbb{R}_{+}^{k} \rightarrow \mathbb{R}$ by

$$
Q(s)=E \Phi\left(\sum_{j=1}^{k} s_{j}^{1 / 2} U_{i}\right)=\int_{\left(S^{n-1}\right)^{k}} \Phi\left(\sum_{j=1}^{k} s_{j}^{1 / 2} x_{j}\right) d x_{1} \ldots d x_{k} .
$$

To prove Theorem 1, we must show that if $a$ and $b$ are points of $\mathbb{R}_{+}^{k}$ with $b=\left(b_{1}, \ldots, b_{k}\right) \prec\left(a_{1}, \ldots, a_{k}\right)=a$, then $Q(a) \leq Q(b)$. Now

$$
\begin{aligned}
Q(b)-Q(a) & =\int_{0}^{1} \frac{d}{d t} Q((1-t) a+t b) d t \\
& =\int_{0}^{1} \sum_{j=1}^{k} \partial_{j} Q((1-t) a+t b)\left(b_{j}-a_{j}\right) d t,
\end{aligned}
$$

where $\partial_{j} Q=\partial Q / \partial s_{j}$. For $i=1, \ldots, k$, set $\Omega_{i}=S^{n-1} \times \ldots \times \mathbb{B}^{n} \times \ldots \times S^{n-1}$, where $\mathbb{B}^{n}$ is the $i$ th factor. Fix $i \in\{1, \ldots, k\}$, integrate in (3.4) first with respect to $x_{i}$, differentiate with respect to $s_{i}$, and apply Lemma 1 to the function $x_{i} \mapsto \Phi\left(x_{i}+\sum_{j \neq i} s_{j}^{1 / 2} x_{j}\right)$. The result is

$$
\partial_{i} Q(s)=\frac{1}{2 n} \int_{\Omega_{i}} \Delta \Phi\left(\sum_{j=1}^{k} s_{j}^{1 / 2} x_{j}\right) d x_{1} \ldots d x_{k}, \quad 1 \leq i \leq k .
$$


Taking $i=1$ in (3.6), and using the relation

$$
\int_{\mathbb{B}^{n}} F(x) d x=\int_{0}^{1} n r^{n-1} d r \int_{S^{n-1}} F(r x) d x,
$$

we obtain

$$
\begin{aligned}
\partial_{1} Q(s) & =\frac{1}{2} \int_{\left(S^{n-1}\right)^{k-2}} d x_{3} \ldots d x_{k} \ldots \\
& \int_{0}^{1} r^{n-1} d r \int_{\left(S^{n-1}\right)^{2}} \Delta \Phi\left(s_{1}^{1 / 2} r x_{1}+s_{2}^{1 / 2} x_{2}+\sum_{j=3}^{k} s_{j}^{1 / 2} x_{j}\right) d x_{1} d x_{2} .
\end{aligned}
$$

Fix the $s_{j}$ and $x_{j}$ for $j \geq 3$, and set $f(z)=\Delta \Phi\left(z+\sum_{j=3}^{k} s_{j}^{1 / 2} x_{j}\right)$. By Lemma 2, the $d x_{1} d x_{2}$ integral in (3.7) equals $I\left(\left|r s_{1}^{1 / 2}+s_{2}^{1 / 2}\right|, f\right)$. Formula (3.7) holds also for $\partial_{2} Q(s)$, provided that $I\left(\left|r s_{1}^{1 / 2}+s_{2}^{1 / 2}\right|, f\right)$ is changed to $I\left(\left|s_{1}^{1 / 2}+r s_{2}^{1 / 2}\right|, f\right)$. If $s_{1} \geq s_{2}$ and $0 \leq r \leq 1$, then

$$
\begin{aligned}
\left|r s_{1}^{1 / 2}+s_{2}^{1 / 2}\right|^{2} & =r^{2} s_{1}+2 r s_{1}^{1 / 2} s_{2}^{1 / 2}+s_{2} \\
& \leq s_{1}+2 r s_{1}^{1 / 2} s_{2}^{1 / 2}+r^{2} s_{2}=\left|s_{1}^{1 / 2}+r s_{2}^{1 / 2}\right|^{2} .
\end{aligned}
$$

Now $f$ is subharmonic on $\mathbb{R}^{n}$, so $I(r, f) \nearrow$ as $r \nearrow$. Thus, we have shown that $s_{1} \geq s_{2} \Rightarrow \partial_{1} Q(s) \leq \partial_{2} Q(s)$. The same argument shows that $s_{i} \geq$ $s_{i+1} \Rightarrow \partial_{i} Q(s) \leq \partial_{i+1} Q(s)$ for each $i=1, \ldots, k-1$. Hence:

$$
\text { If } s_{1} \geq \ldots \geq s_{k} \text {, then } \partial_{1} Q(s) \leq \ldots \leq \partial_{k} Q(s) \text {. }
$$

Let us return now to (3.5). The function $Q$ is permutation invariant, so we may assume that the components of $a$ and of $b$ decrease as $i$ increases. Then for $0 \leq t \leq 1$ the components of $(1-t) a+t b$ also decrease. By (3.8), the sequence $\partial_{j} Q((1-t) a+t b)$ increases as $j$ increases. By assumption, $b \prec a$. If $\lambda=\left(\lambda_{1}, \ldots, \lambda_{k}\right)$ is a length $k$ sequence with increasing terms, it is easy to show, using summation by parts for example, that $b \prec a \Rightarrow$ $\sum_{j=1}^{k} \lambda_{j} b_{j} \geq \sum_{j=1}^{k} \lambda_{j} a_{j}$. Thus, the integrand on the right-hand side of (3.5) is nonnegative, and hence $Q(b) \geq Q(a)$, as required.

Functions $Q$ with the above property, that $b \prec a \Rightarrow Q(a) \leq Q(b)$, are said to be Schur-concave on $\mathbb{R}_{+}^{k}$.

The Gaussian in Corollary 1. With the situation of Corollary 1, we need to verify that

$$
E\left(\left(\Phi_{1}\left(Z_{n}\right)\right)^{-}\right)<\infty
$$

and that

$$
\lim _{k \rightarrow \infty} E \Phi\left(Z_{n, k}\right)=E \Phi_{1}\left(Z_{n}\right) .
$$


If $\Phi$ is continuous and bisubharmonic then so is $\Phi_{1}$. Moreover, for rotationally-invariant $\mathbb{R}^{n}$-valued random variables $W, E|\Phi(W)|<\infty$ if and only if $E\left|\Phi_{1}(W)\right|<\infty$, in which case $E \Phi(W)=E \Phi_{1}(W)$. Thus, to prove (3.9) and (3.10) we may assume that $\Phi$ is itself radial. Set $u=\Delta \Phi$. Then $u$ is radial and subharmonic. Write $u(r)=u\left(r e_{1}\right)$, and $\Phi(r)=\Phi\left(r e_{1}\right)$. Since $u(r)$ is the mean of a subharmonic function on $|x|=r$, it follows that $u$ is finite, continuous and increasing on $(0, \infty)$. Moreover, $\Phi$ satisfies the distributional o.d.e.

$$
r^{1-n} \frac{d}{d r}\left(r^{n-1} \Phi^{\prime}(r)\right)=u(r) .
$$

It is easy to verify that continuous distributional solutions of $(3.11)$ on $(0, \infty)$ are in $C^{2}$. Thus, $\Phi \in C^{2}(0, \infty)$.

Let us assume now that $n \geq 3$. Integrate (3.11) from 1 to $r$. The result is, for $0<r<\infty$,

$$
\begin{aligned}
\Phi(r)= & \Phi(1)+\frac{1}{n-2} \Phi^{\prime}(1)\left(1-r^{2-n}\right) \\
& +\frac{1}{n-2} \int_{1}^{r}\left(1-t^{n-2} r^{2-n}\right) t u(t) d t .
\end{aligned}
$$

Since $u(t) \geq u(1)$ for $t \geq 1,(3.12)$ implies existence of positive constants $a$ and $b$ such that

$$
\Phi(r) \geq-a r^{2}-b, \quad 1 \leq r<\infty .
$$

Since $\Phi$ is continuous on $\mathbb{R}^{n}$, it is bounded below in $|x| \leq 1$, so (3.13) still holds for $r \in[0, \infty)$, with perhaps a larger $b$. The statement (3.9) for $n \geq 3$ follows from (3.13).

If $u$ is bounded above on $(0, \infty)$, then from (3.12) it follows that $|\Phi(r)| \leq$ $a r^{2}+b$ for some constants $a, b$ and all $r \in(0, \infty)$. Then (3.10), for $n \geq 3$, follows from either of the central limit type Theorems 2.2.11 or 2.2.20 in [St 93].

If $u$ is not bounded above, define $v:(0, \infty) \rightarrow \mathbb{R}$ by $u(r)=v\left(r^{2-n}\right)$. A calculation shows that subharmonicity of $u$ is equivalent to convexity of $v$. Moreover, our $v$ is decreasing, and tends to infinity as $r \rightarrow 0$. Let $\left\{r_{m}\right\}$ be a positive sequence in $(0,1)$ which decreases to zero. Set $v_{m}=v$ on $\left[r_{m}, \infty\right)$, and define $v_{m}$ on $\left[0, r_{m}\right]$ to be the linear function with $v_{m}\left(r_{m}\right)=v\left(r_{m}\right)$ and slope equal to the derivative of $v$ from the right at $r_{m}$. Then each $v_{m}$ is convex on $(0, \infty)$, and $v_{m}(r) \nearrow v(r)$ as $m \rightarrow \infty$ for each $r \in(0, \infty)$. Set $u_{m}(r)=v_{m}\left(r^{2-n}\right)$. Define $\Phi_{m}=\Phi$ on $[0,1]$, and on $[1, \infty)$ define $\Phi_{m}$ by replacing $u$ by $u_{m}$ in (3.12). Extend $\Phi_{m}$ to $\mathbb{R}^{n}$ by defining $\Phi_{m}(x)=\Phi_{m}(|x|)$. Then $\left\{\Phi_{m}\right\}$ is a sequence of continuous radial bisubharmonic functions which increases to $\Phi$ pointwise on $(0, \infty)$, and each $\Delta \Phi_{m}=u_{m}$ is bounded above. 
Thus $E \Phi_{m}\left(Z_{n, k}\right) \nearrow E \Phi_{m}\left(Z_{n}\right)$ for each fixed $m$. Hence

$$
E \Phi_{m}\left(Z_{n, k}\right) \leq E \Phi_{m}\left(Z_{n}\right) \leq E \Phi\left(Z_{n}\right) \text { for all } k, m \text {. }
$$

Letting $m \rightarrow \infty$ and applying the Monotone Convergence Theorem, we see that $E \Phi\left(Z_{n, k}\right) \leq E \Phi\left(Z_{n}\right)$ for each $k$, so that $\lim _{k \rightarrow \infty} E \Phi\left(Z_{n, k}\right) \leq$ $E \Phi\left(Z_{n}\right)$. The opposite inequality can be obtained by writing $\Phi=\Phi^{+}-\Phi^{-}$, then applying [St 93, 2.2.2] to $\Phi^{+}$, and $(3.13)$ and [St 93, 2.11] or [St 93, $2.20]$ to $\Phi^{-}$. The proof of (3.10) is complete when $n \geq 3$.

Suppose $n=2$. Then instead of (3.12) we have

$$
\Phi(r)=\Phi(1)+\Phi^{\prime}(1) \log r+\int_{1}^{r}\left(\log \frac{r}{t}\right) t u(t) d t, \quad 1<r<\infty .
$$

The lower bound (3.13) is still true, and hence so is (3.9). If $u$ is bounded above then $u$ is constant, which implies that $\Phi(r)$ has the form $a r^{2}+b$, so that (3.10) is trivial. To handle nonconstant $u$, define $v:(-\infty, \infty) \rightarrow \mathbb{R}$ by $v(x)=$ $u\left(e^{x}\right)$. Then $v$ is convex and increasing. Approximate $v$ by an increasing sequence of convex functions, each of which is linear for sufficiently large $x$. The corresponding approximants to $\Phi$ satisfy bounds of the form $\Phi_{m}(r)=$ $O\left(r^{2} \log r\right)$ as $r \rightarrow \infty$. Theorem 2.2.20 of [St 93] still applies to each $\Phi_{m}$. The proof of (3.10) for $n=2$ can be now accomplished like the one for $n \geq 3$, with a few small changes. A proof of (3.9) and (3.10) for $n=1$ can be constructed along the same lines as for $n=2$.

4. Proof of Theorem 2. Let $\Phi$ be a radial function on $\mathbb{R}^{n}$, with the property that $b \prec a \Rightarrow Q(a) \leq Q(b)$ for each pair of nonnegative $k$-tuples $a, b \in \mathbb{R}_{+}^{k}$, where $Q$ is defined as in (3.4). Assume for now that $\Phi \in C^{4}$. Take $R>0, B \geq 0, \varepsilon>0, k=3, a=\left(R^{2}, 0, B^{2}\right)$, and $b=\left(R^{2}-\varepsilon, \varepsilon, B^{2}\right)$. Then $b \prec a$, so $Q(a)-Q(b) \leq 0$. Dividing by $\varepsilon$ and letting $\varepsilon \rightarrow 0$, we obtain $\partial_{1} Q\left(R^{2}, 0, B^{2}\right)-\partial_{2} Q\left(R^{2}, 0, B^{2}\right) \leq 0$. Set $u=\Delta \Phi$. If we write $x=x_{1}$, $y=x_{3}$, it follows from (3.6) that

$$
\int_{\mathbb{B}^{n} \times S^{n-1}} u(R x+B y) d x d y \leq \int_{S^{n-1} \times S^{n-1}} u(R x+B y) d x d y .
$$

As in the previous section, $d x$ and $d y$ denote uniform probability measures on $\mathbb{B}^{n}$ or $S^{n-1}$, according to the specified domain of integration. From (4.1), (3.3) and a simple computation, we obtain

$$
\int_{\mathbb{B}^{n}} I\left(\left|R x+z_{0}\right|, u\right) d x \leq \int_{S^{n-1}} I\left(\left|R x+z_{0}\right|, u\right) d x,
$$

where $z_{0}$ is any point of $\mathbb{R}^{n}$ with $\left|z_{0}\right|=B$. But $u$ is radial, so $I\left(\left|R x+z_{0}\right|, u\right)=$ $u\left(R x+z_{0}\right)$. Thus, the right-hand integral in (4.2) equals the mean value of $u$ over the sphere $\left|x-z_{0}\right|=R$. Similarly, the left-hand integral equals the 
mean over the ball $\left|x-z_{0}\right| \leq R$. We have thus shown that for every $z_{0} \in \mathbb{R}^{n}$ and every $R>0$,

$$
\int_{\mathbb{B}^{n}} u\left(z_{0}+R x\right) d x \leq \int_{S^{n-1}} u\left(z_{0}+R x\right) d x .
$$

Now $u \in C^{2}\left(\mathbb{R}^{n}, \mathbb{R}\right)$. If $u$ were not subharmonic, there would exist $z_{0} \in$ $\mathbb{R}^{n}$ such that $\Delta u\left(z_{0}\right)<0$. Then $u$ would be strictly superharmonic in some ball centered at $z_{0}$, the spherical mean on the right in (4.3) would be a strictly decreasing function of $R$ for small $R$, and the left side would be strictly larger than the right side for small $R$. This contradiction shows that $u$ must be subharmonic, so that $\Phi$ must be bisubharmonic. Theorem 2 is proved for radial $\Phi \in C^{4}$.

Let now $\Phi$ be radial, continuous and satisfy the hypotheses of Theorem 2 . Take $x_{0} \in \mathbb{R}^{n}$, and set $\Psi(x)=\Phi\left(x+x_{0}\right), B=\left|x_{0}\right|$. By converting to integrals over products of spheres, or otherwise, one may show that

$$
E \Psi\left(\sum_{i=1}^{k} a_{i} U_{i}\right)=E \Phi\left(\sum_{i=1}^{k} a_{i} U_{i}+B U_{k+1}\right)
$$

for sequences $\left(a_{1}, \ldots, a_{k}\right)$, where $U_{k+1}$ is uniformly distributed on $S^{n-1}$ and is independent of $U_{1}, \ldots, U_{k}$. If $\left(b_{1}^{2}, \ldots, b_{k}^{2}\right) \prec\left(a_{1}^{2}, \ldots, a_{k}^{2}\right)$, then $\left(b_{1}^{2}, \ldots, b_{k}^{2}, B^{2}\right) \prec\left(a_{1}^{2}, \ldots, a_{k}^{2}, B^{2}\right)$. Since $(1.1)$ holds for $\Phi$ and the augmented sequences, it holds also for $\Psi$ and the original sequences. Consequently, for any nonnegative integrable compactly supported function $K$ on $\mathbb{R}^{n}$, if $\left\{b_{i}^{2}\right\} \prec\left\{a_{i}^{2}\right\}$ then (1.1) holds with $\Phi$ replaced by the convolution $K * \Phi$. If we take $K$ to be also $C^{\infty}$ and radial, then $K * \Phi$ is $C^{\infty}$ and radial, so the first part of the proof implies that $K * \Phi$ is bisubharmonic. Standard arguments, like those in [Hö 94, p.148], imply that $\Phi$ is bisubharmonic.

5. Proof of Theorem 3. The usual smoothing methods work straightforwardly in this situation, so we will only consider the case when $\Phi \in$ $C^{4}\left(\mathbb{R}^{n}, \mathbb{R}\right)$. Fix $x_{0} \in \mathbb{R}^{n}$, and set

$$
h(t)=\int_{S^{n-1}} \Phi\left(x_{0}+t^{1 / 2} x\right) d x, \quad u(x)=\Delta \Phi\left(x_{0}+x\right) .
$$

Lemma 1, with $f(x)=\Phi\left(x+x_{0}\right)$, implies that $h^{\prime}(t)$ is $1 /(2 n)$ times the mean value of $u$ over the ball $\left|x-x_{0}\right|<t^{1 / 2}$, so that

$$
h^{\prime}(t)=\frac{1}{2} t^{-n / 2} \int_{0}^{t^{1 / 2}} I(s, u) s^{n-1} d s=\frac{1}{2 n} t^{-n / 2} \int_{0}^{t^{n / 2}} I\left(r^{1 / n}, u\right) d r .
$$

If $\Phi$ is bisubharmonic, then $I(s, u) \nearrow$ as $s \nearrow$, so $h^{\prime}(t) \nearrow$ as $t \nearrow$, hence $h$ is convex. Conversely, if the $C^{4}$ function $\Phi$ were not bisubharmonic, there would exist $x_{0} \in \mathbb{R}^{n}$ such that $\Delta \Delta \Phi(x)<0$ in some neighborhood of $x_{0}$. 
The corresponding $u$ would be strictly superharmonic in a ball around the origin, $I(s, u)$ would strictly decrease for all small $s$, and (5.1) would show that $h^{\prime}(t)$ strictly decreases for all small $t$. Thus, $h$ would not be convex.

6. Sharp Khinchin inequalities for balls. Let $V_{1}, \ldots, V_{k}$ be independent $\mathbb{R}^{n}$-valued random variables, each of which is uniformly distributed on the unit ball $\mathbb{B}^{n}$ of $\mathbb{R}^{n}$. We continue to let $a_{1}, \ldots, a_{k}$ denote a real sequence of $k$ elements. H. König [Kö 98], [KK 01] discovered the following remarkable identity:

KÖNIG's IDEnTITY. For $n \geq 1$,

$$
E\left|\sum_{i=1}^{k} a_{i} V_{i}\right|^{p}=\frac{n}{n+p} E\left|\sum_{i=1}^{k} a_{i} U_{i}\right|^{p}, \quad 0<p<\infty,
$$

where $U_{1}, \ldots, U_{k}$ are independent $\mathbb{R}^{n+2}$-valued random variables, each of which is uniformly distributed on $S^{n+1}$.

This identity permits deduction of sharp $L^{2}-L^{p}$ Khinchin inequalities for balls from the corresponding inequalities for spheres. For example, using (1.6), one obtains, for $n \geq 1, p \geq 2$ and $\sum_{i=1}^{k} a_{i}^{2}=1$,

$$
\frac{n}{n+p} \leq E\left|\sum_{i=1}^{k} a_{i} V_{i}\right|^{p} \leq E\left|k^{-1 / 2} \sum_{i=1}^{k} V_{i}\right|^{p} \leq \frac{n}{n+p} E\left|Z_{n+2}\right|^{p} .
$$

The quantities on the right and left ends are the smallest constants which work for all $k$ and fixed $n$. For $n=1$, these inequalities, and the reverse inequalities for $1 \leq p \leq 2$, were first proved by Latała and Oleszkiewicz [LO 95].

We shall state a $\Phi$ moment generalization of König's identity. For simplicity, we confine attention to radial moments. Thus, let $\Phi$ be a continuous radial function on $\mathbb{R}^{n}$. Then $\Phi(x)=\Phi_{1}(|x|)$ for a continuous function $\Phi_{1}$ on $[0, \infty)$. Define $\Psi_{1}:(0, \infty) \rightarrow \mathbb{R}$ by

$$
\Psi_{1}(r)=n r^{-n} \int_{0}^{r} \Phi_{1}(s) s^{n-1} d s, \quad r \geq 0,
$$

and define a radial function $\Psi$ on $\mathbb{R}^{n+2}$ by $\Psi(x)=\Psi_{1}(|x|)$.

Theorem 4. Let the $U_{i}$ and $V_{i}$ be as in König's identity. Then

$$
E \Phi\left(\sum_{i=1}^{k} a_{i} V_{i}\right)=E \Psi\left(\sum_{i=1}^{k} a_{i} U_{i}\right)
$$

From Theorem 4, one sees that the inequalities (1.1) and (1.2) of Theorem 1 and Corollary 1 hold when $X=\sum_{i=1}^{k} a_{i} U_{i}, Y=\sum_{i=1}^{k} b_{i} U_{i}$, and $Z_{n, k}$ are replaced by $\sum_{i=1}^{k} a_{i} V_{i}, \sum_{i=1}^{k} b_{i} V_{i}$, and $k^{-1 / 2} \sum_{i=1}^{k} V_{i}$, provided $\Phi$ 
is radial and $\Psi$ is bisubharmonic on $\mathbb{R}^{n+2}$. The reader may verify that a smooth radial $\Phi$ has biharmonic $\Psi$ if and only if $\Phi_{1}$ satisfies the differential inequality

$$
r^{2} \Phi_{1}{ }^{\prime \prime \prime}(r)+\left(n^{2}-n\right)\left(r \Phi_{1}{ }^{\prime \prime}(r)-\Phi_{1}{ }^{\prime}(r)\right) \geq 0, \quad 0<r<\infty .
$$

Proof of Theorem 4. Let $f$ and $g$ denote the density functions of $\left|\sum_{i=1}^{k} a_{i} U_{i}\right|$ and $\left|\sum_{i=1}^{k} a_{i} V_{i}\right|$, respectively. We claim that

$$
f(r)=-\frac{r^{n}}{n}\left(r^{1-n} g(r)\right)^{\prime} .
$$

Assuming (6.5), and using also (6.2), we have

$$
\begin{aligned}
E \Psi\left(\sum_{i=1}^{k} a_{i} U_{i}\right) & =\int_{0}^{\infty} \Psi_{1}(r) f(r) d r=-\int_{0}^{\infty} \Psi_{1}(r) \frac{r^{n}}{n}\left(r^{1-n} g(r)\right)^{\prime} d r \\
& =n^{-1} \int_{0}^{\infty}\left(r^{n} \Psi_{1}(r)\right)^{\prime} r^{1-n} g(r) d r=\int_{0}^{\infty} \Phi_{1}(r) g(r) d r \\
& =E \Phi\left(\sum_{i=1}^{k} a_{i} V_{i}\right) .
\end{aligned}
$$

To complete the proof of the theorem, we must verify (6.5). To do this, we use some ideas from a proof of (6.1) in [KK 01, p. 126] which is attributed to Latała. Its point of departure is the observation that if $U$ is uniform on $S^{n+1}$ and $V$ is uniform on $\mathbb{B}^{n}$, then $U \cdot e_{1}$ and $V \cdot e_{1}$ have the same distribution, given by the density $c_{n}\left(1-t^{2}\right)^{(n-1) / 2}$ for $|t|<1$ and suitable constant $c_{n}$.

Let $F$ and $G$ denote the density functions, defined on $\mathbb{R}^{n+2}$ and $\mathbb{R}^{n}$, respectively, of $\sum_{i=1}^{k} a_{i} U_{i}$ and $\sum_{i=1}^{k} a_{i} V_{i}$. Then $F$ and $G$ are radial, and so are their Fourier transforms $\widehat{F}$ and $\widehat{G}$. With the normalizations of [SW 71], we have

$$
\begin{aligned}
\widehat{F}\left(r e_{1}\right) & =E\left(\exp \left(-2 \pi i r e_{1} \cdot \sum_{i=1}^{k} a_{i} U_{i}\right)\right) \\
& =E\left(\exp \left(-2 \pi i r e_{1} \cdot \sum_{i=1}^{k} a_{i} V_{i}\right)\right)=\widehat{G}\left(r e_{1}\right), \quad r \geq 0 .
\end{aligned}
$$

Write $H(r)=\widehat{F}\left(r e_{1}\right)=\widehat{G}\left(r e_{1}\right)$, and define, for $t \geq 0, n \geq 2$,

$$
P_{n}(t)=\int_{S^{n-1}} e^{2 \pi i t e_{1} \cdot x} d x=c \int_{0}^{\pi} e^{2 \pi i t \cos \theta} \sin ^{n-2} \theta d \theta .
$$

Here, and below, $c$ will denote a constant depending on $n$ which can change from identity to identity. When $n=2$ the term $\sin ^{n-2} \theta$ should be replaced by the constant 1 . When $n=1$, define $P_{1}(t)=\cos 2 \pi t$. 
The Fourier inversion formula and conversion to polar coordinates [SW 71, pp. 11, 154, 155] give

$$
\begin{aligned}
& F\left(r e_{1}\right)=\int_{\mathbb{R}^{n+2}} \widehat{F}(\xi) e^{2 \pi i r e_{1} \cdot \xi} d \xi=c \int_{0}^{\infty} P_{n+2}(t r) H(t) t^{n+1} d t, \\
& G\left(r e_{1}\right)=\int_{\mathbb{R}^{n}} \widehat{G}(\xi) e^{2 \pi i r e_{1} \cdot \xi} d \xi=c \int_{0}^{\infty} P_{n}(t r) H(t) t^{n-1} d t .
\end{aligned}
$$

Differentiation, then integration by parts in the last integral in (6.7), leads to $P_{n}^{\prime}(t)=-c t P_{n+2}(t)$. Using this with (6.8), we deduce

$$
\frac{\partial G}{\partial r}\left(r e_{1}\right)=-c r F\left(r e_{1}\right) \text {. }
$$

The density functions $f, F, g, G$ are related by

$$
f(r)=c F\left(r e_{1}\right) r^{n+1}, \quad g(r)=c G\left(r e_{1}\right) r^{n-1} .
$$

From (6.9) and (6.10), it follows that $f(r)=-c r^{n}\left(r^{1-n} g(r)\right)^{\prime}$. Using $\int_{0}^{\infty} f(r) d r=\int_{0}^{\infty} g(r) d r=1$, one can show that the constant $c$ in the last identity equals $1 / n$. The proof of (6.5), and of Theorem 4 , is complete.

The function $P_{n}$ is expressible in terms of Bessel functions:

$$
P_{n}(t)=\frac{2 \pi}{\omega_{n-1}} t^{-(n-2) / 2} J_{(n-2) / 2}(2 \pi t)
$$

where $\omega_{n-1}$ is the area of $S^{n-1}$. See, for example, [SW 71, p. 154].

Acknowledgements. We are grateful to P. Hitczenko for telling us about the paper [LO], to R. Latała and K. Oleszkiewicz for helpful communications, to A. Pełczyński for calling the attention of H. König to the results in [C 98], and to R. Rochberg for telling us about [NP 96]. We thank especially Professors König and Kwapień for sending us their papers, and for valuable comments.

\section{References}

[C 98] R. C. Culverhouse, Sharp majorization inequalities for sums of independent uniformly distributed random variables, thesis, Washington University, 1998.

[E 70] M. L. Eaton, A note on symmetric Bernoulli random variables, Ann. Math. Statist. 41 (1970), 1223-1226.

[Fa 87] S. Yu. Favorov, Distribution of values of holomorphic mappings of $\mathbb{C}^{m}$ into a Banach space, Funktsional. Anal. i Prilozhen. 21 (1987), no. 3, 91-92 (in Russian); English transl.: Functional Anal. Appl. 21 (1987), 251-252.

[Fa 98] - A generalized Kahane-Khinchin inequality, Studia Math. 130 (1998), 101-107.

[F 97] T. Figiel, P. Hitczenko, W. B. Johnson, G. Schechtman and J. Zinn, Extremal properties of Rademacher functions with applications to the Khintchine and Rosenthal inequalities, Trans. Amer. Math. Soc. 349 (1997), 997-1027. 
[GF 87] E. A. Gorin and S. Yu. Favorov, Generalizations of Khinchin's inequality, Teor. Veroyatnost. i Primenen. 35 (1990), 763-767 (in Russian); English transl.: Theory Probab. Appl. 35 (1990), 766-771.

[Ha 82] U. Haagerup, The best constants in the Khintchine inequality, Studia Math. 70 (1982), 231-283.

[Hö 94] L. Hörmander, Notions of Convexity, Birkhäuser, Boston, 1994.

[Kh 23] A. Ya. Khinchin, Über dyadische Brüche, Math. Z. 18 (1923), 109-116.

[Kö 98] H. König, On the best constants in the Khintchine inequality for variables on spheres, preprint, Universität Kiel, 1998.

[KK 01] H. König and S. Kwapien, Best Khintchine type inequalities for sums of independent, rotationally invariant random vectors, Positivity 5 (2001), 115-152.

[Kom 88] R. Komorowski, On the best possible constants in the Khintchine inequality for $p \geq 3$, Bull. London Math. Soc. 20 (1988), 73-75.

[LO 95] R. Latała and K. Oleszkiewicz, A note on sums of independent uniformly distributed random variables, Colloq. Math. 68 (1995), 197-206.

[MO 79] A. Marshall and I. Olkin, Theory of Majorization and its Applications, Academic Press, New York, 1979.

[NP 96] F. L. Nazarov and A. N. Podkorytov, Ball, Haagerup, and distribution functions, preprint, 1996, http://www.math.msu.edu/ fedja/prepr.html.

[Pe 85] A. Pełczyński, Norms of classical operators in function spaces, Astérisque 131 (1985), 137-162.

[Peš 95] G. Peškir, Best constants in Kahane-Khintchine inequalities for complex Steinhaus functions, Proc. Amer. Math. Soc. 123 (1995), 3101-3111.

[S 85] J. Sawa, The best constant in the Khintchine inequality for complex Steinhaus functions, Studia Math. 81 (1985), 107-126.

[Ste 61] S. B. Stechkin, On the best lacunary system of functions, Izv. Akad. Nauk SSSR Ser. Mat. 25 (1961), 357-366 (in Russian).

[SW 71] E. Stein and G. Weiss, Introduction to Fourier Analysis on Euclidean Spaces, Princeton Univ. Press, Princeton, 1971.

[St 93] D. Stroock, Probability Theory, an Analytic View, Cambridge Univ. Press, Cambridge, 1993.

[Sz 76] S. J. Szarek, On the best constant in the Khintchine inequality, Studia Math. 58 (1976), 197-208.

[U 88] D. C. Ullrich, Khinchin's inequality and zeros of Bloch functions, Israel J. Math. 62 (1988), 56-62.

[W 60] P. Whittle, Bounds for the moments of linear and quadratic forms in independent random variables, Theory Probab. Appl. 5 (1960), 302-305.

[Y 76] R. M. G. Young, On the best constants in the Khintchine inequality, J. London Math. Soc. 14 (1976), 496-504.

Mathematics Department

Washington University

St. Louis, MO 63130, U.S.A.

E-mail: al@math.wustl.edu
Department of Psychiatry Washington University Medical School St. Louis, MO 63110, U.S.A. E-mail: rob@frodo.wustl.edu 